\title{
A INTERNET DAS COISAS COM AS TECNOLOGIAS RFID E NFC
}

\author{
Victor Nassar \\ Universidade Federal de Santa Catarina \\ victornassar@gmail.com \\ Milton Luiz Horn Vieira \\ Universidade Federal de Santa Catarina \\ milton.vieira@ufsc.br
}

\begin{abstract}
Resumo: O crescimento tecnológico propicia uma série de oportunidades à sociedade, sejam em áreas financeiras, em educação, entretenimento, entre outros. Ao mesmo tempo, pode auxiliar em dificuldades costumeiras, como trânsito, segurança e saúde. Nesse cenário, a tecnologia está inserida ao participar da modificação do modo de vida das pessoas, na maneira como consomem e produzem informações, e em como se relacionam entre si e com o meio. Cada vez mais, diferentes dispositivos atuam em uma relação não apenas entre os indivíduos, mas também com outros aparelhos, enviando dados e conectando-se entre si, configurando assim a internet das coisas. Entre as tecnologias presentes nesta conexão entre objetos, destacam-se o RFID (Radio Frequency Identification) e o NFC (Near Field Communication). Assim, este artigo aborda a influência da internet das coisas na sociedade, através de um estudo analítico que traz aplicações em diferentes modalidades, como pagamentos móveis, acesso a conteúdos digitais, games, transporte, entre outros. Por fim, geram-se discussões sobre o papel da internet das coisas no auxílio à realização de tarefas, no relacionamento entre as pessoas e em como pode proporcionar diferentes formas de entretenimento.
\end{abstract}

Palavras-chave: Design de Interação, Internet das Coisas, RFID (Radio Frequency-Identification), NFC (Near Field Communication).

\footnotetext{
Abstract: The technological growth provides a number of opportunities to society, whether in financial, consumer and entertainment, for example. At the same time, can assist in customary difficulties, such as traffic, safety and health. In this scenario, technology is inserted to participate in the modification of the way of life of the people, the way assimilate and produce information, and also how they relate to each other and with the environment. Increasingly, different devices operate in a relationship not only between people, but also with other objects by sending data and connecting with each other, thus setting the internet of things. Among the
} 
technologies presented on this connection between objects, we highlight the RFID (Radio Frequency Identification) and NFC (Near Field Communication). Thus, this paper discusses the influence of internet of things in society, through an analytical study that brings applications in different modalities, such as mobile payments, access to digital content, games, transportation, among others. Finally, discussions are generated on the role of Internet of things in helping to carry out tasks, in the relationship between people and how they can provide different forms of entertainment.

Key-words: Interaction Design, Internet of Things, RFID (Radio FrequencyIdentification), NFC (Near Field Communication).

\section{INTRODUÇÃO}

A evolução da tecnologia móvel tem contribuído para o desenvolvimento de um cenário altamente conectado, explorando as características do ser humano na busca por socialização e acesso a informações, em qualquer lugar, tempo e dispositivo. Cada vez mais presente no cotidiano das pessoas, a plataforma mobile é ampliada para uma crescente integração entre diferentes dispositivos e produtos, como carros, rádios e eletrônicos. É esta realidade em que variados objetos podem conectar-se sem fio à internet e entre si que configura a chamada "Internet das Coisas" (Internet of Things IOT) (ATZORI, IERA \& MORABITO, 2010).

O NFC (Near Field Communication) e o RFID (Radio Frequency Identification), por exemplo, são tecnologias integradas a aparelhos eletrônicos que podem influenciar a experiência dos usuários em variadas aplicações, possibilitando a realização de pagamentos, o acesso a catracas, a obtenção e transmissão de conteúdo (OK et. al., 2011).

Nesse contexto, este artigo apresenta um estudo analítico com aplicações que utilizam as tecnologias NFC e RFID e que demonstram como a internet das coisas está sendo incorporada na sociedade. Destaca-se a importância das aplicações no sentido de como a tecnologia pode ajudar a solucionar ou minimizar os problemas urbanos, assim como auxiliar na realização de tarefas diárias e proporcionar diferentes formas de entretenimento.

O artigo inicia com a fundamentação teórica na seção 2 sobre o conceito de internet das coisas, seguindo na seção 3 com a contextualização das tecnologias RFID e NFC. Após, tem-se a seção 4. Estudo Analítico, com a aplicação das tecnologias RFID e NFC em modalidades comumente encontradas para a interação, divididas em: 4.1 Cartão de acesso, 4.2 Pagamentos Móveis, 4.3 Tags e 4.4 Transferência de dados entre dispositivos. Por fim, levantam-se questionamentos de como a internet das coisas pode influenciar a sociedade, nas relações humanas e entre dispositivos.

\section{A INTERNET DAS COISAS}

Internet das Coisas (Internet of Things -loT) é um conceito apresentado em 1999, com a intenção primordial de servir de expressão aos objetos materiais que estavam ligados à internet (SUN, 2012). A partir de então, a internet das coisas vem ganhando terreno no cenário de telecomunicações sem fios. A idéia básica deste 
conceito é a presença generalizada de uma variedade de coisas ou objetos - como Radio-Frequency Identification (RFID), sensores, telefones celulares, etc - que, através de esquemas de endereçamento único, são capazes de interagir uns com os outros e cooperar com seus vizinhos para alcançar objetivos comuns (GIUSTO et. al., 2010).

Presser e Gluhak (2009) já apontavam tecnologias como pilares da internet das coisas, como RFID, NFC (Near Field Communication), redes wireless e sensores sem fio. No entanto, também destacavam que um amplo portfólio de dispositivos, redes e serviços de tecnologia construiriam a internet das coisas, unindo o mundo real ao digital. Não obstante, é importante enfatizar no conceito de que a internet das coisas deve ser focada em "coisas". Conforme Sterling (2005), a partir do momento em que há um aumento de objetos inteligentes, que podem ser rastreados e que criam uma comunicação entre si, é que abre-se caminho para a implantação concreta da internet das coisas.

Dessa forma, pode-se entender a configuração a partir de quaisquer itens que estejam ligados entre si, para a troca de informação e comunicação, a fim de identificar, localizar, rastrear, monitorar e gerenciar uma rede. É uma realidade em que a tecnologia consolida-se como importante aliada do ser humano para a realização de tarefas diárias, a partir da massiva utilização de smartphones, notebooks, GPS, redes $\mathrm{Wi}-\mathrm{Fi}$, tablets e outros eletrônicos.

Assim, destaca-se que a principal força da ideia internet das coisas está no alto impacto que pode ter sobre vários aspectos do cotidiano e comportamento dos usuários. Do ponto de vista de um usuário particular, os efeitos mais óbvios da internet das coisas está na introdução nos campos de trabalho e na vida doméstica. Da mesma forma, encontra-se utilização em áreas da indústria e comerciais, como automação, logística, gestão, transporte, publicidade. A partir da exploração e avanço da tecnologia, a perspectiva inclui elementos de saúde, educação, segurança e demais aspectos de benefícios comuns na sociedade.

Neste contexto, diferentes tecnologias são consideradas como elementoschave para a consolidação da internet das coisas. Com objetos de estudo deste artigo, destacam-se a seguir as definições das tecnologias RFID e NFC, que servirão de base para o entendimento das aplicações apresentadas no estudo analítico realizado.

\section{CONTEXTUALIZANDO O RFID E O NFC}

A seguir, são apresentadas definições e características das tecnologias RFID e NFC, bem como modos de utilização e o procedimento particular de como é realizada a comunicação dos dados em cada uma.

\subsection{Definição do RFID}

O RFID é uma rede de comunicação à distância sem fio, que funciona pela de identificação de frequências de rádio, com alcance até aproximadamente $10 \mathrm{~m}$, de acordo com o chip utilizado. A comunicação ocorre através de uma etiqueta com chip RFID, a chamada Tag RFID, que envia sinais a um leitor específico. A partir disso, um software é responsável pela conversão dos dados em informações significativas (DUROC \& KADDOR, 2012).

Há dois modos de funcionamento de uma etiqueta RFID. No modo Ativo, a Tag possui uma fonte de alimentação através de uma bateria e são capazes de enviar 
dados a um leitor por conta própria. Já no modo passivo, não há bateria e a corrente é fornecida pelo leitor.

Desse modo, a tecnologia RFID pode ser utilizada com o objetivo de identificação ou rastreamento de objetos, aplicações do setor logístico, de supermercados, transporte ou cargas. Apenas é necessário que o produto possua a etiqueta com RFID e múltiplos dados podem ser capturados de uma vez pelo leitor, mesmo que os produtos estejam em movimento, sem a necessidade de contato ou visão do produto (FREIBERGER \& BEZERRA, 2010; NAVARRO, GRILLO \& LIMA, 2008; SOARES et al. 2008; SUN, 2012).

\subsection{Definição do NFC}

Diferentemente do RFID, o NFC (Near Field Communication) é uma tecnologia que permite a transferência de dados em uma comunicação sem fio de curta distância. O NFC surgiu a partir do RFID, por isso muitos dos benefícios observados anteriormente são compartilhados por ambas as tecnologias. Foi desenvolvido pela Sony e Philips em 2002 e impulsionado a partir de 2004 pelo NFC Forum, que é conduzido por empresas como Samsung, Microsoft, Nokia, Google, Intel e Visa (NFC FORUM, 2011)

Assim como o RFID, a comunicação acontece de maneira simples e intuitiva, apenas aproximando os dispositivos com NFC. Dessa forma, os usuários podem realizar transações financeiras, acessar conteúdos digitais ou conectar diferentes aparelhos. Os chips também podem vir tanto em smartphones e eletrônicos, quanto em etiquetas (Tags NFC/RFID). Com isso, é possível estabelecer a comunicação em diferentes objetos físicos, como crachás, chaveiros e cartões.

As aplicações com NFC podem ser categorizadas em três tipos de operação: a) Reader/Writer: um dispositivo com NFC identifica dados em uma Tag NFC; b) Card Emulation: um dispositivo com NFC simula o comportamento de um cartão, para a realização de transações financeiras ou acesso a catracas e portas; c) Peer-to-Peer: dois dispositivos são emparelhados, estabelecendo uma comunicação entre ambos (OZDENIZCl et. al., 2010). Ressalta-se que o RFID também executa as operações de acesso a catracas, identificação de Tags e emparelhamento de dispositivos. Porém, não realiza-se transações financeiras com RFID, pela distância maior na comunicação não oferecer a mesma segurança, em relação ao NFC.

\section{ESTUDO ANALÍTICO: APLICAÇÕES DA INTERNET DAS COISAS COM RFID E NFC}

Para a etapa de estudo analítico, foram coletadas aplicações que utilizavam as tecnologias RFID e NFC, executando assim a internet das coisas. Utilizou-se como parâmetro para a categorização das aplicações, as modalidades mais comumente encontradas para a interação, definidas nas seções: 4.1 Cartão de acesso, 4.2 Pagamentos Móveis, 4.3 Tags e 4.4 Transferência de dados entre dispositivos. Procurou-se apresentar através do estudo analítico, uma diversificação de funcionamento, com RFID e NFC oferecendo variadas soluções em fins de praticidade e entretenimento.

\subsection{Cartão de acesso}

Como visto anteriormente, tanto o NFC quanto o RFID podem transformar objetos em chaves codificadas e únicas de acesso, que podem ser utilizadas para abrir 
uma porta de um quarto de hotel, liberar catracas ou mesmo ligar um aparelho. A Hyundai já lançou até mesmo um protótipo de um carro que substitui as tradicionais chaves por um sistema com NFC. A função também é utilizada como ingresso ou bilhete eletrônico, em que o usuário compra a entrada pelo smartphone e apresenta no terminal do evento em questão. A seguir, detalham-se projetos que utilizam as tecnologias como cartões de acesso para auxílio no transporte.

\subsubsection{NFC em programa de ônibus Roda SP}

O projeto Roda SP é realizado durante o verão em São Paulo e dá direito a circular por $24 \mathrm{~h}$ pelos municípios da baixada Santista com uma única passagem de ônibus. Assim, o passageiro pode subir e descer entre os 32 pontos participantes quantas vezes quiser. $O$ controle era realizado até $2012 \mathrm{com}$ uma pulseira simples no braço, o que dificultava o controle da Secretaria de Turismo. Em 2013, o programa testou o Cartão de Acesso NFC, desenvolvido pelo CPqD. Ao comprar a passagem, 0 usuário recebia um cartão NFC, que era utilizado em cada embarque e desembarque dos ônibus. Ao passar o cartão na primeira parada, as $24 \mathrm{~h}$ de uso começavam a contar. A aplicação foi feita associada a smartphones presentes nos veículos e não em catracas. Dessa forma, o sistema com NFC contribuiu com a logística do Roda SP e os relatórios da Secretaria, que teve o controle exato do número de passageiros que estiveram nos ônibus, os pontos com maior fluxo e os períodos mais intensos de utilização (NFC BRASIL, 2013).

\subsubsection{RFID em acesso a pedágios no Brasil}

Desde 2000, algumas cidades no Brasil possuem o sistema de pedágio Sem Parar/Via Fácil que possibilita o acesso inteligentes de veículos por catracas nas estradas. O projeto funciona com o usuário fixando a Tag RFID no automóvel. Ao se aproximar do local do pedágio, a cancela identifica a Tag e libera a passagem, sem a necessidade de parar o carro. Ao final do mês, o usuário recebe o boleto com o valor dos pedágios. Em 2013, o sistema sofreu algumas modificações com o intuito de tornar o valor do pedágio segmentado de acordo com a quantidade de quilômetros trafegados (RFID JOURNAL BRASIL, 2013a).

O sistema Ponto a Ponto ainda passa por testes e funciona de maneira similar ao Sem Parar, mas com o modelo de pré-pagamento. Assim, o usuário compra créditos que são descontados conforme a utilização dos pedágios. O programa também instalou pontos com leitores RFID pelas estradas, que funcionam para identificar as etiquetas dos carros, para que a cobrança seja feita de acordo com a distância percorrida e sem a necessidade de catracas (RFID JOURNAL BRASIL, 2013b).

\subsection{Pagamentos Móveis}

Área na qual se encontram grandes investimentos, com a possibilidade de realizar transações financeiras, substituindo os cartões de crédito. Para isso, o usuário precisa de um aplicativo específico para cadastrar o seu cartão, realizando a transação aproximando um celular do outro ou de um terminal de pagamento e confirmando com a senha.

Em 2011, teve inicio o Google Wallet nos EUA e em 2012 começou a ser utilizado o PagSeguro NFC no Brasil. Já em 2013, as empresas de telefonia Claro, Vivo e Tim começaram a oferecer o serviço de pagamento móvel com o apoio dos bancos 
Bradesco e Itaú, além das empresas MasterCard e Visa. Segundo pesquisa da Exame (2013), a empresa de terminais de pagamento Cielo também atualizou mais de 600 mil equipamentos com NFC no Brasil em 2013. Também se concretizou o sistema PayWave da Samsung internacionalmente.

Desde 2007, são realizados testes para a realização de pagamentos com NFC substituindo os tradicionais bilhetes de passagem utilizados em transporte público. Em cidades da Alemanha, o projeto Touch\&Travel testou com aproximadamente 3 mil participantes a aceitação do NFC em smartphones como meio de acesso para o checkin e check-out em trens. Os benefícios informados foram a flexibilidade para a emissão de bilhetes, com baixa infraestrutura e custo.

Nos EUA, uma parceria entre o sistema de trânsito de São Francisco e empresas de fast-food resultou em um projeto que possibilitava o pagamento móvel com NFC no transporte público e ainda a compra de refeições nos estabelecimentos parceiros através de smart posters presentes nas estações de embarque. Da mesma forma, em Londres também foi testado um projeto que utilizava smartphones com NFC para compra de bilhetes e acesso, em substituição aos cartões de transporte tradicionais. Como resultado, os participantes demonstraram alto nível de interesse e satisfação no projeto, pela conveniência, facilidade de uso e status que sentiram com a aplicação (NFC FORUM, 2011).

\subsection{Tags RFID/NFC}

Utilizada para a distribuição de conteúdo ou para habilitar funções específicas no celular e em diferentes equipamentos eletrônicos. Uma Tag RFID/NFC é como uma etiqueta que possui o chip RFID ou NFC. Dentre as possibilidades, pode-se integrar o chip em diversos objetos, como pulseiras, chaveiros, cartazes e cartões. Além disso, o chip também pode estar presente em equipamentos eletrônicos, como aparelhos de música, televisores, computadores, entre outros, atuando de forma ativa para acionar funções predeterminadas.

\subsubsection{Tags diversificadas para interações}

Além das etiquetas gerais comercializadas, existem as etiquetas das próprias empresas de smartphones, como a "Sony Smart Tags" e a "LG Tags", que podem habilitar funções do celular apenas ao aproximá-lo das etiquetas. Entre as funções, pode-se utilizar para acionar o GPS quando a pessoa estiver no carro ou oferecer o acesso ao Wi-Fi de um evento sem a necessidade de inserir a senha ou precisar deixálo como sinal público, uma vez que a própria etiqueta efetua o trabalho de login.

No evento Coca-Cola Village, realizado em 2010, cada participante recebia uma pulseira com RFID na entrada, que era associada ao seu perfil no Facebook. Assim, em cada local de diversão do evento, como áreas de piscinas, shows e restaurantes, havia uma estação para que o participante aproximasse sua pulseira e que automaticamente compartilhava um conteúdo no Facebook (RFID JOURNAL, 2010).

Outro exemplo é o StartCap, uma tampa de cerveja com RFID. O chip vem integrado há um dispositivo de movimento na tampa de metal. Ao abrir a garrafa, há a ativação do lacre desenvolvido, que envia o sinal de RFID e inicia uma ação previamente determinada. Assim, foi utilizada em eventos-testes nos quais, ao abrir a garrafa, iniciava funções, como: tocar uma música no ambiente, acender um painel luminoso próximo e compartilhar conteúdo nas redes sociais (START CAP, 2014). 


\subsubsection{Smart posters}

Os smart posters são cartazes que possuem a etiqueta e tem sido utilizado pela publicidade e em eventos. Basta aproximar o aparelho do local indicado no pôster e a pessoa recebe o conteúdo. Com finalidade cultural, uma biblioteca pública virtual foi criada na Áustria, disseminando adesivos NFC que possibilitavam o download de livros. O projeto consiste em aplicar smart posters em diferentes locais públicos. Ao aproximar o smartphone do cartaz, a pessoa poderá escolher entre alguns livros que estão à disposição e efetuar o download da obra.

Outro exemplo é o do festival de curtas Go Short na Holanda. Os cartazes de divulgação vinham com Tags NFC e QR-Codes, além de uma breve instrução ao público, mostrando como deveriam fazer para interagirem (ver Figura 1). Ao aproximar o celular do local indicado, o usuário recebias informações sobre os próximos filmes que seriam exibidos no Festival e os locais em que estariam passando. Toda a ação faz ainda mais sentido pela cidade em que foi realizada. Além da Holanda ser considerada um dos pólos de inovação em tecnologia, user experience e design, Nijmegen é uma grande cidade estudantil, com uma agitada vida noturna e grande aposta em comunicação jovem e irreverente (GO SHORT, 2013).

E é seguindo este caminho que a agência de marketing The Picture Works comandou a divulgação dos filmes O Cavaleiro Solitário, com Johnny Depp, e The Way Way Back, com Steve Carell. Assim, parte da campanha apresentava cartazes dos filmes com a incorporação das etiquetas com NFC (ver Figura 1). Os smart posters foram disponibilizados em bares e restaurantes da Irlanda. Os cartazes ficavam em locais especiais, com uma iluminação destacada. Ao aproximar o celular do espaço indicado no pôster, as pessoas tinham acesso ao trailer no YouTube (THE PICTURE WORKS, 2013)
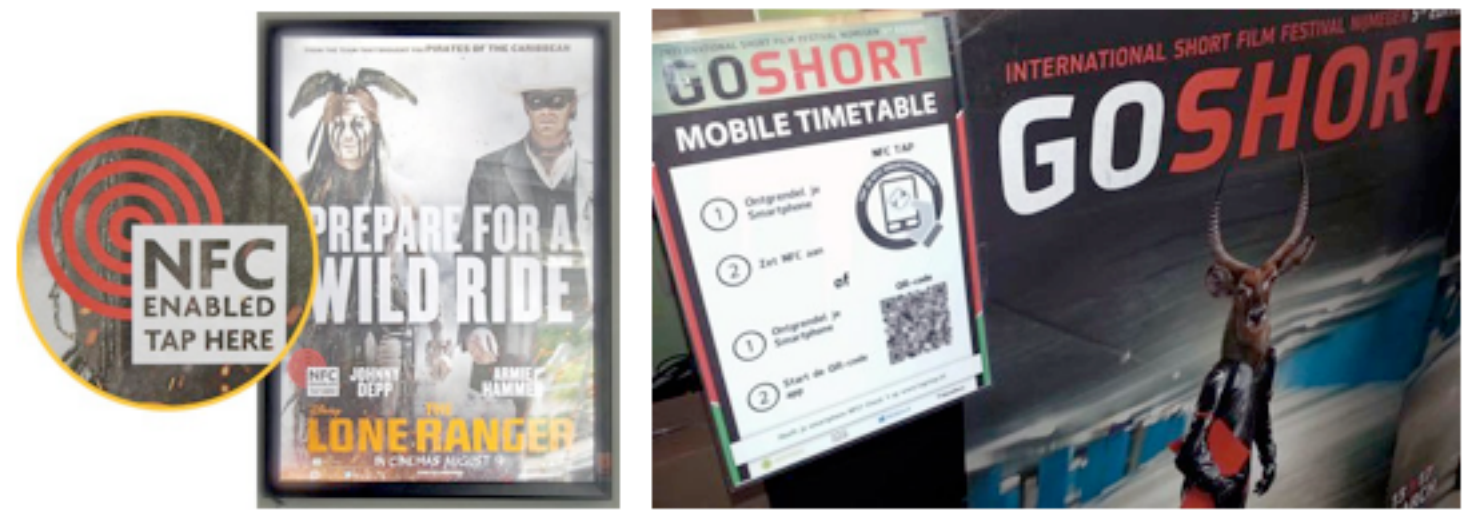

Figura 1 - Smart Posters do filme O Cavaleiro Solitário e do Festival Go Short.

Fonte: Adaptado de The Pictures Works (2013) e Go Short (2013).

Na edição especial do Lollapalooza, a Billboard Brasil trouxe um conceito de revista inteligente, com uma capa atuando como um smart poster, integrando um chip NFC. Na capa vinha uma instrução para a pessoa colocar seu smartphone e ao aproximar o aparelho do local, recebia uma lista com músicas da banda. A ideia da Billboard era fazer com que uma revista de música toque música, sem a necessidade de um QR-Code ou download, apenas usando a tecnologia NFC e seu celular para a reprodução do som (ADNEWS, 2013). 


\subsubsection{Smart table}

Uma smart table atua como um smart poster, integrando uma Tag em locais determinados em uma mesa. Como parte da campanha de divulgação do filme "Thor: O Mundo Sombrio", a Marvel, em parceria com a empresa Insert, utilizou mesas com chips RFID/NFC para criar interações com o público (ver Figura 2). A ideia ocorreu em cafés e restaurantes na França, que tinham suas mesas personalizadas com adesivos sobre o filme e vinham com as etiquetas por baixo do vidro. Ao aproximar o smartphone com NFC do local especificado, o usuário tinha acesso a um aplicativo móvel do filme, que possibilitava participar de uma competição para ganhar ingressos, além do acesso ao trailer e do compartilhamento de conteúdo no Facebook e Twitter (NFC WORLD, 2013a).

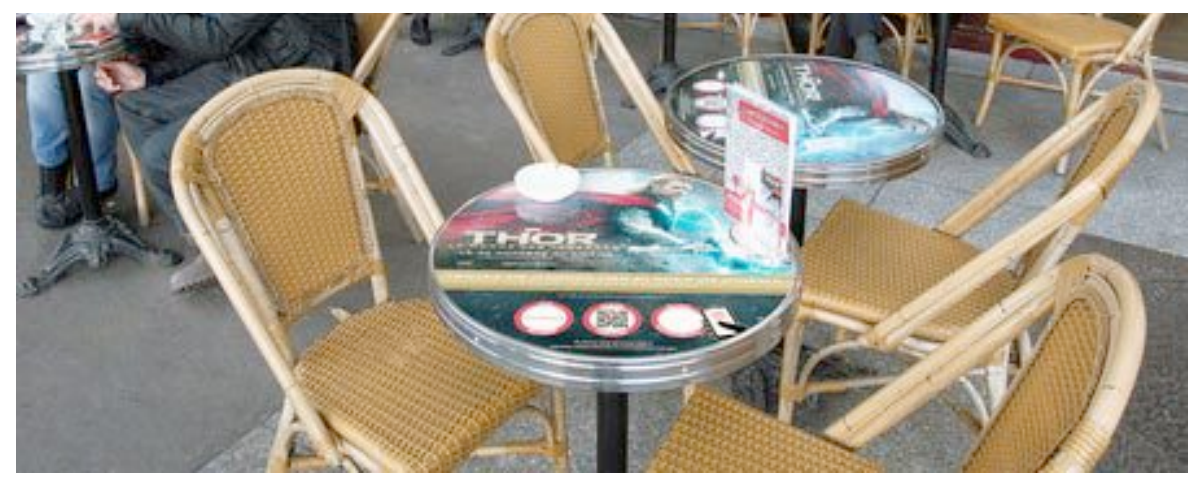

Figura 2 - A mesa com NFC para o filme Thor: O Mundo Sombrio. Fonte: NFC World (2013a).

\subsubsection{Games}

A indústria dos jogos também vislumbra utilizar o NFC para expandir a experiência de jogabilidade e produzir diferentes integrações entre objetos nos games. O novo jogo de Pokemon que a Nintendo anunciou para o console Wii U terá aplicação com bonecos reais através de NFC (ver Figura 3). A iniciativa representa o começo de uma série de novas funcionalidades que videogame terá, principalmente com a incorporação da tecnologia NFC no controle GamePad. Basta aproximar a miniatura ao local indicado no controle e o personagem automaticamente é habilitado no jogo (NINTENDO, 2013).

Outras utilizações também tem sido observadas, como a inclusão de Tags NFC em jogos de cartas e criando uma interação em que o jogador devia aproximar o smartphone da carta para receber uma missão ou ver se ganhou um bônus.

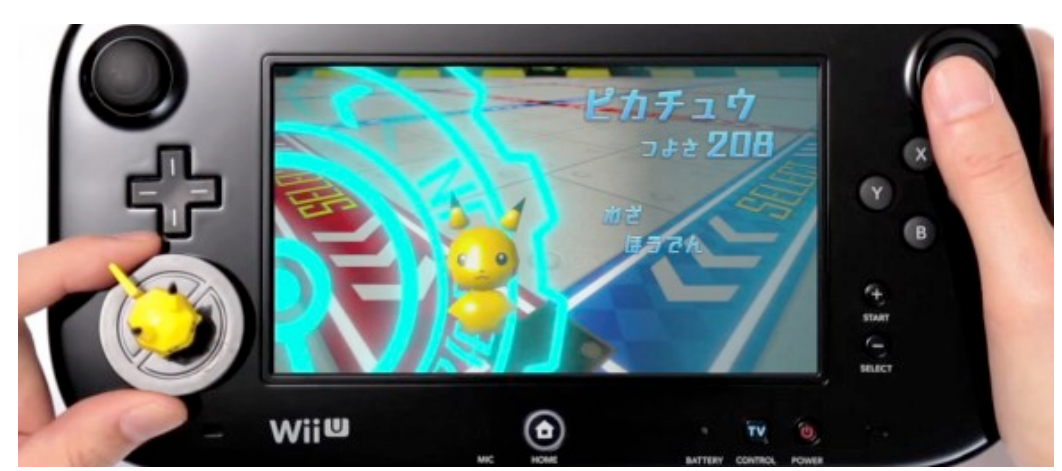

Figura 3 - Nintendo Wii U integra o boneco no jogo Pokemon. Fonte: Nintendo (2013). 


\subsection{Transferência de dados entre dispositivos}

A tradicional transferência de dados entre aparelhos, tal como acontece com o envio de um foto ou música por um bluetooth ou uma rede Wi-Fi. A diferença está no emparelhamento entre os aparelhos e na facilidade de enviar e receber os dados. A transferência também não precisa ser apenas entre telefones e pode ser efetuada entre TVs, Tablets, Notebooks ou qualquer outro objetos, desde que habilitados com chips ativos de RFID ou NFC, capazes de enviar e receber dados.

\subsubsection{Câmera com NFC}

A Sony lançou uma nova câmera digital, equipada com as tecnologias Wi-Fi e NFC. O modelo compacto NEX-5T vem com 16.1 megapixel e é a primeira câmera da Sony a apresentar NFC, oferecendo assim a possibilidade de transferência e compartilhamento de fotos e vídeos para outros aparelhos com a tecnologia (NFC WORLD, 2013b). Desse modo, a Sony coloca em prática sua ideia de conectar seus eletrônicos, a partir da inclusão de NFC em suas TVs, Smartphones (Linha Xperia), Notebooks (Sony Vaio) e outros periféricos. A estratégia é semelhante a adotada pela LG, ao lançar a SmarTv com NFC e divulgar o projeto para a casa inteligente com eletrodomésticos com NFC.

\subsubsection{Eletrodomésticos da casa do futuro}

$\mathrm{Na}$ feira de tecnologia CES que aconteceu em Janeiro de 2013 em Las Vegas, a LG apresentou o projeto Smart Home Services. Utilizando a tecnologia NFC nos eletrodomésticos e eletrônicos, a empresa oferece uma comunicação inteligente de controle pelos smartphones (LG NEWS, 2013). Assim, o conceito de internet das coisas é colocado em prática com a possibilidade real de estar inserido no hábito familiar em um futuro próximo.

Pelo que foi apresentado na CES 2013, a LG implementou e desenvolveu aplicações de NFC para os mais diversos produtos de utilidades do lar, como Geladeira, Máquina de Lavar, Fogão, Microondas e Aspirador de Pó (ver Figura 4). A ideia do MagicRemoto e do SmartControl é que o consumidor possa não apenar ligar e desligar os eletro-eletrônicos pelo smartphone, mas também possuir um histórico de funcionamento, conferir o tempo que resta para as roupas terminarem de lavar ou para um bolo ficar pronto no forno, otimizando o tempo em casa. Além disso, com o auxílio de outras conexões, o usuário poderia dar um comando de voz para os aparelhos pelo celular (LG NEWS, 2013).

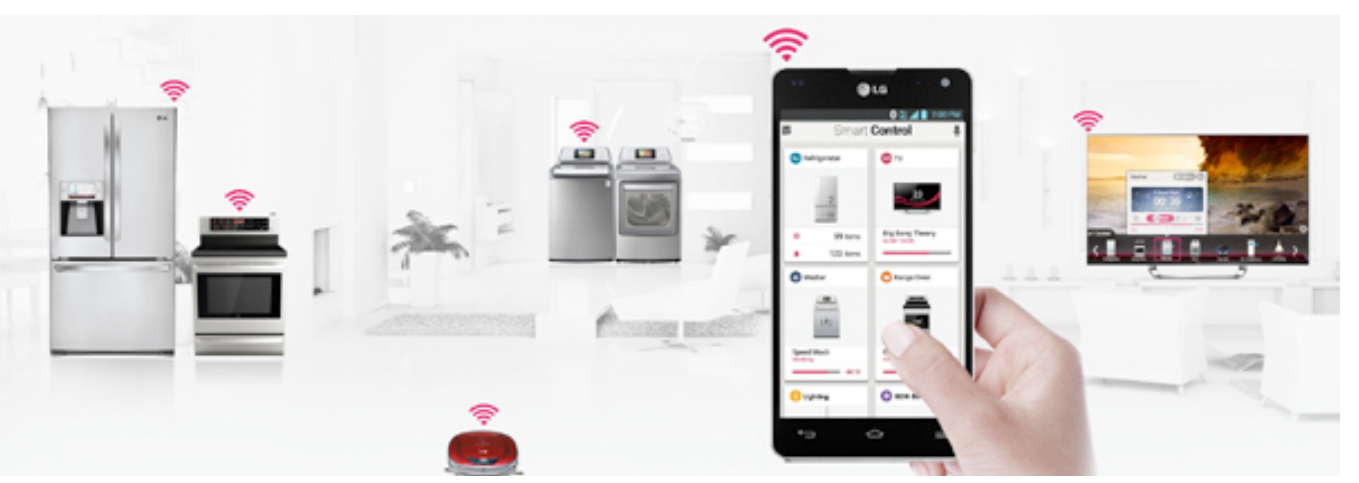

Figura 4 - Eletrodomésticos e eletrônicos configurando a internet das coisas.

Fonte: LG NEWS (2013). 
Já em específico para as TVs, de acordo com LG NEWS (2103), a LG desenvolveu o SmartShare, no qual os espectadores poderão transferir conteúdo entre diferentes dispositivos. A aplicação funciona com uma associação entre o NFC e uma rede Wi-Fi ou 3G/4G. Aproximando o smartphone da TV, o NFC ativa instantaneamente o conteúdo a ser compartilhado e a transferência seria executada pelas outras redes. A ideia é deixar a tecnologia preparada, já esperando o futuro idealizado para a TV Digital, com o consumidor realizando na hora o pagamento de um produto que acabou ver na programação

\section{DISCUSSÃO}

A crescente integração entre variados dispositivos propicia um mundo hiperconectado, visando à resolução de diferentes atividades cotidianas. Assim, o poder internet das coisas está nos benefícios que pode trazer para as pessoas, através da incorporação na sociedade. Com o aumento da aplicação de tecnologias como RFID e NFC em áreas culturais, comerciais, de transporte e gestão, há uma perspectiva positiva para a consolidação da internet das coisas, mesmo que em longo prazo.

Ações como a da utilização de bibliotecas virtuais, que são consultadas através de smartphones por diferentes pontos das cidades, atraem público e posicionam um foco para a cultura, podendo oferecer um novo conceito de acesso ao conhecimento e da educação. Em termos de mercado em si, a experiência para a internet das coisas ganha ainda mais alcance, em virtude de trabalhar com consumidores que anseiam por inovação.

No meio cinematográfico, a incorporação das tags NFC em cartazes de filmes, ampliam a experiência publicitária para o público, uma vez que, além de imagem, pode oferecer um extenso conteúdo sobre a produção, como sinopse, site e trailer. Com isso, o marketing podia atingir o público pertinente ao filme e ainda oferecer um conteúdo diretamente em seus aparelhos. Outra vantagem de se utilizar locais fechados é a possibilidade de ter também rede Wi-Fi disponível, ampliando o acesso à web e evitando a não-visualização por falta de internet.

Embora possa parecer distante, o compartilhamento de conteúdo de uma TV para a internet já é uma realidade. Além da proliferações de SmarTVs com acesso à rede, novas relações também são observadas em aparelhos com o PlayStation 4 da Sony, no qual os jogadores podem compartilhar imediatamente vídeos e fotos do jogo, além de poder realizar ações em diferentes plataformas (extensão do jogo em celular e na web) para o console, como parte da jogabilidade.

O projeto da Nintendo com NFC também pode representar um avanço tecnológico significativo para indústria dos games, somando ao ideal de compartilhamento e integração propostos pelo PlayStation 4. Com a aceitação do público para estas iniciativas, poderá ser ainda mais comum ver aplicações diferentes entre NFC e os jogos, ampliando o conceito do que representa um jogo.

Neste contexto, vê-se que a aplicação das internet das coisas na "casa do futuro" idealizada pela LG não se encontra em uma realidade distante e pode ser ainda mais forte para os próximos anos. E com a inclusão das redes sociais nesse processo, torna-se ainda mais interessante vislumbrar as possibilidades de interação entre pessoas e objetos. A ação da Coca-Cola e a pulseira do "Like" com RFID estiveram na vanguarda, mas é recente a tampa de cerveja com RFID que é capaz de realizar 
diferentes ações. Com simples Tags RFID/NFC, por exemplo, tudo se torna passível de um "Curtir", estando a um passo do Facebook.

A interação entre dispositivos começa a criar novas relações, intensificadas pela capacidade de comunicação diversificada e troca de dados. Indo um pouco mais adiante, mas não tão longe, é possível visualizar um compartilhamento de um filme que acabou de ver no cinema, uma fase que acabou de passar num videogame ou uma comida que acabou de ficar pronta em um fogão. Tudo isso podendo ser realizado ao aproximar o smartphone da poltrona em que estava sentado, da conexão do console com a internet e do eletrodoméstico com um "smartwatch". Assim, tantos "objetos inteligentes" tendem a surgir, alterando não apenas a relação entre o ser humano e suas coisas, mas nas relações com o mundo de um modo geral.

Observa-se a mudança nas formas de gerenciar e operacionalizar aspectos da educação, trânsito, saúde, economia, publicidade, entre outras áreas influentes na sociedade. Mesmo em relações básicas diárias em casa e momentos de lazer. Uma conversa em uma festa poderá acontecer de um novo modo, por exemplo. A pessoa vai querer adicionar a outra em sua rede de contatos só aproximando o celular. Quase uma cantada: posso encostar meu smartphone no seu? A única desculpa será a de que acabou a bateria. Por enquanto.

Nesse contexto, é um exercício estimulante idealizar diferentes aplicações para a internet das coisas, simplesmente envolvendo relações entre o meio e objetos. E não precisa nenhuma ficção para vislumbrar a concretização disso. O contexto começa a ser modificado em uma clara alteração no modo como os indivíduos estão assimilando e produzindo informações. Assim, a internet das coisas ganha forma não apenas em conceito, pois a realidade é de afirmação de grandes projetos e de empresas poderosas, capazes de ditar tendências de consumo e comportamento na sociedade.

\section{REFERÊNCIAS}

ADNEWS. Turning magazines into music players. 2013. Disponível em: <http://www.adnews.com.au/adnews/turning-magazines-into-music-players >. Acesso em 10 ago. 2013.

ATZORI, L.; IERA, A.; MORABITO, G. The Internet of Things: A survey. Computer Networks v.54. pp. 2787-2805. 2010

DUROC, Y.; KADDOUR, D. RFID potential impacts and future evolution for Green projects. In: Energy Procedia, Elsevier/Science Direct, vol. 18, 2012, pp.91-98.

EXAME. Cielo é a empresa vencedora no setor de serviços. 2013. Disponível em: < http://exame.abril.com.br/negocios/noticias/cielo-e-a-empresa-vencedora-no-setorde-servicos>. Acesso em: 12 jul. 2013.

FREIBERGER, A.; BEZERRA, M. B. P. RFID e Seus Impactos na Logística. Março, 2010. Disponível em: <http://www.logisticadescomplicada.com/rfid-e-seus-impactos-nalogistica/>. Acesso em 14 ago. 2013.

GO SHORT. International Short Film Festival Nijmegen. 2013. Dinsponível em: <http://www.goshort.nl/>. Acesso em: 15 dez. 2013.

GUISTO, D. et. al. The Internet of Things. Springer, 2010. 
LG NEWS. LG Electronics ushers in new age of smart home convergence at CES 2013. 2013. Disponível em:

$<$ http://Ignewsroom.com/ces2013/view.php?category=86\&product_code=135\&produ ct_type=135\&post_index=2954>. Acesso em 15 dez. 2013.

PRESSER, M.; GLUHAK, A. The Internet of Things: Connecting the Real World with the Digital World, EURESCOM mess@ge - The Magazine for Telecom Insiders, vol. 2, 2009.

NAVARRO, C.; GRILLO, A.; LIMA, R. Análise e Proposição de Melhorias no Processo Logístico em uma Multinacional de Tecnologia de Informação e Automação. Anais do XXVIII Encontro Nacional de Engenharia de Produção. Rio de Janeiro-RJ: Outubro 2008.

NFC WORLD. Marvel promotes Thor with NFC Tables. 2013a. Disponível em: <http://www.nfcworld.com/2013/11/20/326949/marvel-promotes-thor-nfc-tables/> Acesso em 20 dez. 2013.

Sony unveils NFC-enabled Nex compact câmera. 2013b. Disponível em: <http://www.nfcworld.com/2013/08/28/325622/sony-unveils-nfc-enabled-nexcompact-camera/>. Acesso: 30 ago. 2013.

NINTENDO. First Pokémon Game exclusively for Wii U, Pokémon Ruble U, lauching august 29 via Nintendo eShop. 2013. Disponível em:

$<$ http://www.nintendo.com/whatsnew/detail/FuXMGHORuz-9nz8dwaB2hxCqlOdvbxb> Acesso em 15. Dez. 2013.

NFC BRASIL. Projeto de São Paulo testa NFC em ônibus da Baixada Santista. 2013. Disponível em: <http://nfcbrasil.wordpress.com/2013/01/18/projeto-de-sao-paulotesta-nfc-em-onibus-da-baixada-santista/> Acesso em 12 ago. 2013.

OK, K. et. al. Exploring Underlying Values of NFC Applications. 3rd International Conf. on Information and Financial Engineering. IPEDR v12, 2011.

OZDENIZCI, B. et. al. NFC Research Framework: A Literature Review And Future Research Directions, Proc. 14th IBIMA International Business Information Management Conf., pp. 2672-2685.Istanbul, TURKEY, 2010.

THE PICTURES WORKS. Digital Augmented Ink. 2013. Disponível em: <http://thepictureworks.eu/projects/34>. Acesso em 13 nov. 2013.

RFID JOURNAL. RFID Helps Make Friends for Israeli Teens. 2010. Disponível em: <http://www.rfidjournal.com/articles/view?7830>. Acesso em 20 jan.2014.

RFID JOURNAL BRASIL. Sistema Sem Parar/Via Fácil para pagamento de pedágios terá upgrade em 2013. 2013a. Disponível em:

<http://brasil.rfidjournal.com/noticias/vision?9320/1>. Acesso em 12 dez. 2013. . Projeto com RFID pode tornar o pedágio mais justo nas estradas de São Paulo. 2013b. Disponível em: $<$ http://brasil.rfidjournal.com/noticias/vision?9662/2>. Acesso em 12 dez. 2013. START CAP. About the Start Cap. 2014. Disponível em: <http://www.start-cap.com/>. Acesso 13. Fev. 2014.

STERLING, B. Shaping Things Mediawork Pamphlets. The MIT Press, 2005. 
SOARES, R. S. et. al. O Impacto da Tecnologia de Etiqueta Inteligente (RFID) na Performance de Cadeias de Suprimentos - Um Estudo no Brasil. Revista Jovens Pesquisadores, ano V, n. 9, jul./dez. 2008.

SUN, C. Application of RFID Technology for Logistics on Internet of Things. AASRI Conference on Computational Intelligence and Bioinformatics, 2012. 